\title{
Isolation of Chemical Compounds and Essential Oil from Agrimonia asiatica Juz. and Their Antimicrobial and Antiplasmodial Activities
}

\author{
Raushan A. Kozykeyeva (D), ${ }^{1,2}$ Ubaidilla M. Datkhayev ${ }^{(D)}{ }^{1}$ \\ Radhakrishnan Srivedavyasasri ${ }^{D}{ }^{2}$ Temitayo O. Ajayi $\mathbb{D}^{2,3}$ \\ Anapiya K. Patsayev $\mathbb{D}^{4}{ }^{4}$ Raikhan A. Kozykeyeva $\mathbb{D}^{5}{ }^{5}$ \\ and Samir A. Ross $\left(D^{2,6}\right.$ \\ ${ }^{1}$ Department of Organization and Management and Economics of Pharmacy and Clinical Pharmacy, School of Pharmacy, \\ Asfendiyarov Kazakh National Medical University, Almaty, Kazakhstan \\ ${ }^{2}$ Center for Natural Product Research, School of Pharmacy, University of Mississippi, Oxford, MS 38677, USA \\ ${ }^{3}$ Department of Pharmacognosy, Faculty of Pharmacy, University of Ibadan, Ibadan, Nigeria \\ ${ }^{4}$ Laboratory of Biochemistry, M. Auezov South Kazakhstan State University, Shymkent, Kazakhstan \\ ${ }^{5}$ Department of Chemistry, South Kazakhstan State Pedagogical University, Shymkent, Kazakhstan \\ ${ }^{6}$ Department of Biomolecular Sciences, Division of Pharmacognosy, School of Pharmacy, University of Mississippi, Oxford, \\ MS 38677, USA
}

Correspondence should be addressed to Raushan A. Kozykeyeva; nar_rau@mail.ru

Received 5 December 2019; Revised 14 February 2020; Accepted 25 February 2020; Published 30 March 2020

Academic Editor: Ghadir A. El-Chaghaby

Copyright (C) 2020 Raushan A. Kozykeyeva et al. This is an open access article distributed under the Creative Commons Attribution License, which permits unrestricted use, distribution, and reproduction in any medium, provided the original work is properly cited.

Agrimonia asiatica is a perennial plant with deep green color and covered with soft hairs and has a slightly aromatic odor. This genus Agrimonia has been used in traditional medicines of China, Greece, and European countries. It was mainly used as a haemostatic, a tonic for asthenia, and an astringent for diarrhea. Agrimony is part of the division Magnoliophyta; class is represented by order Rosales, family Rosaceae, of the genus Agrimonia. Family Rosaceae-or pink eels-is one of the largest families of flowering plants, including about 100 genera and 3000 species. Rosaceae is common in almost all areas of the globe where flowering plants can grow, but most of them are concentrated in the temperate and subtropical zones of the Northern Hemisphere. Phytochemical investigation on ethanolic extract of $A$. asiatica led to isolation of four flavonoid derivatives (kaempferol-3-glycoside, quercetin-3-O- $\alpha$-arabinofuranosyl- $\beta$-D-galactopyranoside, 3-O-kaempherol 2,3-di-O-acetyl-4-O-(cisp-coumaroyl)-6-O-(trans-p-coumaroyl)- $\beta$-D-glucosopyranoside, and catechin) alongside of sucrose. All the extracts, fractions, and isolated compounds were tested for antimicrobial and antiplasmodial activities. We also studied the chemical composition of essential oil obtained from the aerial part of $A$. asiatica. The essential oil constituents from the aerial part of $A$. asiatica were obtained using a steam-distillation method in wild growing conditions in Kazakhstan. The essential oil extracted from the aerial part of the plant was analyzed by gas chromatography-mass spectroscopy and its major components amounting to $100 \%$ were found to be $\beta$-selinene (36.370\%), $\alpha$-panasinsene (21.720\%), hexadecanoic acid (7.839\%), and 1,2-nonadiene (6.199\%). Neither the extract nor the isolated compounds showed antimicrobial and antiplasmodial activities.

\section{Introduction}

Asian agrimony (Agrimonia asiatica Juz.) is a perennial herb reaching a height of up to $140 \mathrm{~cm}$, rhizomatous, erect stem, densely dressed, like leaf stalks, long, stiff hairs with an admixture of shorter and softer hairs. The leaves are green above, below grayish-green, broken-pinnate, from top to bottom slightly pubescent, velvety-pubescent with a mixture of small pieces of iron. The flowers are yellow, collected in spike-shaped inflorescences. The fruits are drooping; the 
TABLE 1: Chemical compounds isolated from Agrimonia asiatica ethanolic extract.

$\begin{gathered}\text { Compound } \\ \text { no. } \\ \text { instrument }\end{gathered}$
Name of compound
Exact mass: 636.1479

2

DMSO/400

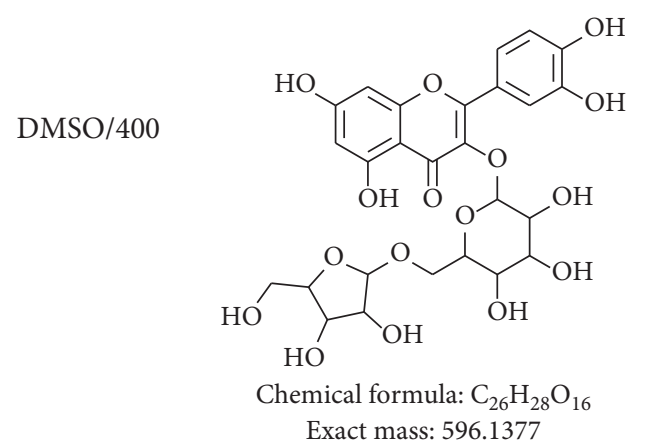

Quercetin-3-O- $\alpha$-arabinofuranosyl- $\beta$-D-galactopyranoside [11]

Kaempferol-3-glycoside [12]

3

DMSO/400<smiles>O=c1c(OC2OC(CO)C(O)C(O)C2O)c(-c2ccc(O)cc2)oc2cc(Cl)cc(O)c12</smiles>

Chemical formula: $\mathrm{C}_{21} \mathrm{H}_{20} \mathrm{O}_{11}$ Exact mass: 448.1006

4

DMSO/400<smiles>Oc1cc(O)c2c(c1)OC(c1ccc(O)c(O)c1)C(O)C2</smiles>

Catechin [13]

Chemical formula: $\mathrm{C}_{15} \mathrm{H}_{14} \mathrm{O}_{6}$ Exact mass: 290.0790 
TABLE 1: Continued.

\begin{tabular}{|c|c|c|c|}
\hline $\begin{array}{l}\text { Compound } \\
\text { no. }\end{array}$ & $\begin{array}{c}\text { Solvent/ } \\
\text { instrument }\end{array}$ & Structure & Name of compound \\
\hline
\end{tabular}

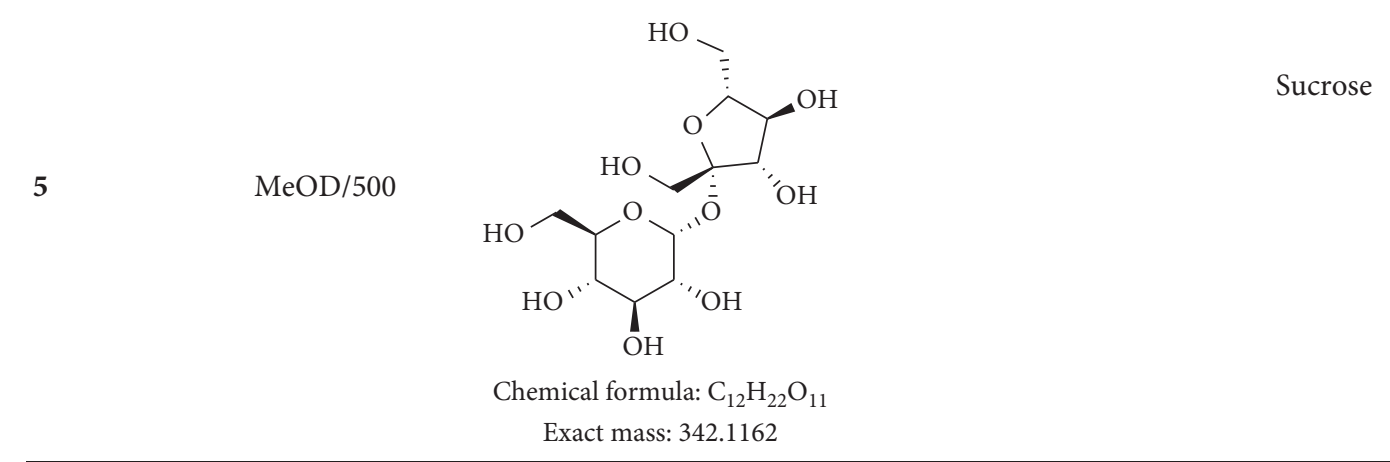

upper surface of the fruit is convex, with spines arranged in several rows, of which the outer spines are shorter than the inner ones. A. asiatica is rich in polysaccharides and glycosides which grows in Central Asia and widely used in folk medicine to prevent and cure diseases connected with gastrointestinal tract, in high blood pressure, and as immunostimulant, astringent. It grows on the slopes of mountains, ditches, gardens, along roads, and on the edges of birch forests, walnut forests. It blooms in June-July. Phytochemical composition of Agrimonia genus includes mainly polysaccharides up to $20 \%$ and tannins in the range of $3.1-10.8 \%$ [1]. In Kazakhstan, A. asiatica extracts were found to have antioxidant activity [2]; suppression of plasma GR activity and an alloxan-induced increase in erythrocyte GP activity were encountered [3]. Previous investigation on essential oil composition of this genus plants A. eupatoria, A. pilosa wild leaves were reported to have caryophyllene, caryophyllene oxide, humulene, and E-farnesene, and in the cultivated leaves oil $\alpha$-pinene, $\beta$-caryophyllene, E- $\beta$-farnesene, and cumin aldehyde were found $[4,5]$. However, this is the first report on essential oil composition from this plant. In continuation to our previous work on essential oil composition of Kazakhstan medicinal plants [6], we were interested to continue working on A. asiatica. Agrimoniin was first isolated in 1982 from roots of $A$. pilosa, a plant traditionally used in Japan and China as an antidiarrheal, haemostatic, and antiparasitic agent. Agrimoniin is a constituent of medicinal plants, which are often applied orally in the form of infusions, decoctions, or tinctures. It is also present in commonly consumed food products, such as strawberries and raspberries. It is metabolized by human gut microbiota into a series of low-molecular-weight urolithins with proven anti-inflammatory and anticancer in vivo and in vitro bioactivities. Agrimoniin is an important component of plants belonging to the Rosaceae family, which have been used since antiquity in traditional medicine to treat a variety of ailments. Owing to a series of highly interesting activities described in detail, agrimoniin is an attractive research candidate for a full exploration of its therapeutic effects [7].

\section{Materials and Methods}

2.1. Plant Material. Aerial parts with seeds of Agrimonia asiatica were collected from Kaskasu village in the Turkestan region in Kazakhstan in June 2018, on the slopes of the mountains, and identified by Dr. N. G. Gemejiyeva (Institute of Botany and Phytointroduction, Science CommitteeMinistry of Education and Science of the Republic of Kazakhstan). A voucher specimen (No. 01-08/3) was deposited in the Herbarium of the Research Institute of Botanical Garden, Almaty, Kazakhstan. The plant material was dried in room at temperature $25^{\circ} \mathrm{C}$ supplied with ventilation. After drying, the raw material was chopped till 3-4 mm using laboratory mill IKA M20.

2.2. Isolation. The chopped plant material $(0.850 \mathrm{~kg})$ was airdried at room temperature. Plant material was first defatted using hexane and then extraction was by percolation in $96 \%$ ethanol ( $4 \mathrm{~L})$ for $24 \mathrm{~h}$ to obtain ethanol extract $(36.926 \mathrm{~g})$. The ethanol extract was adsorbed in celite on a Silica gel column and eluted with solvent system (EtOAc:DCM: $\mathrm{MeOH}: \mathrm{H}_{2} \mathrm{O}(10: 6: 4: 1), 100 \% \mathrm{MeOH}$, and $10 \% \mathrm{MeOH}$ : $\mathrm{H}_{2} \mathrm{O}$. All the fractions were monitored using TLC and pooled into 10 main fractions. Main fraction $1(8.534 \mathrm{~g})$ was adsorbed in silica $(8.00 \mathrm{~g})$ and placed in a silica gel column and eluted with DCM:MeOH mixtures in increasing polarities to obtain 4 subfractions. Subfraction $1(0.5 \mathrm{~g})$ was loaded onto a Sephadex LH-20 column and eluted with $10 \%$ $\mathrm{MeOH}$ :DCM. 15 fractions were collected, from which fraction 14 was identified as 3-O-kaempherol 2,3- di-O-acetyl-4O-(cis-p-coumaroyl)-6-O-(trans-p-coumaroyl)- $\beta$-D-glucosopyranoside). Subfraction $4(1.258 \mathrm{~g})$ was loaded onto a Sephadex LH-20 column and eluted with $\mathrm{MeOH}$ and 16 subfractions were collected. Subfraction 9 was identified as kaempferol-3-glycoside; subfraction 11 was identified as catechin. Main fraction $4(1.2891 \mathrm{~g})$ was loaded onto a Sephadex LH-20 column and eluted with $\mathrm{MeOH}$; yielded nine subfractions were collected. Subfraction 5 was identified as 
TABLE 2: NMR data for compound 1 in DMSO- $d_{6}$.

\begin{tabular}{|c|c|c|}
\hline C. no. & ${ }^{13} \mathrm{C}$ NMR $(126 \mathrm{MHz}) \delta$ & {$[10]$} \\
\hline 2 & 156.53 & 156.3 \\
\hline 3 & 132.93 & 132.7 \\
\hline 4 & 177.24 & 177.1 \\
\hline 5 & 161.15 & 161.2 \\
\hline 6 & 98.89 & 98.7 \\
\hline 7 & 164.53 & 164.2 \\
\hline 8 & 93.77 & 93.7 \\
\hline 9 & 156.40 & 156.6 \\
\hline 10 & 103.77 & 103.9 \\
\hline $1^{\prime}$ & 120.64 & 120.6 \\
\hline $2^{\prime}, 6^{\prime}$ & 130.85 & 130.8 \\
\hline $3^{\prime}, 5^{\prime}$ & 115.17 & 115.1 \\
\hline $4^{\prime}$ & 160.09 & 160.1 \\
\hline $1^{\prime \prime}$ & 100.78 & 98.5 \\
\hline $2^{\prime \prime}$ & 71.94 & 74.2 \\
\hline $3^{\prime \prime}$ & 77.11 & 73.7 \\
\hline $4^{\prime \prime}$ & 67.79 & 70.0 \\
\hline $5^{\prime \prime}$ & 73.89 & 74.1 \\
\hline $6^{\prime \prime}$ & 62.58 & 62.8 \\
\hline $8^{\prime \prime \prime}$ & 166.18 & 166.1 \\
\hline $7^{\prime \prime \prime}$ & 113.58 & 113.6 \\
\hline $6^{\prime \prime \prime}$ & 144.75 & 144.7 \\
\hline $1^{\prime \prime \prime}$ & 124.93 & 124.9 \\
\hline $2^{\prime \prime \prime}, 6^{\prime \prime \prime}$ & 130.23 & 130.2 \\
\hline $3^{\prime \prime \prime}, 5^{\prime \prime \prime}$ & 115.77 & 115.8 \\
\hline $4^{\prime \prime \prime}$ & 159.86 & 159.8 \\
\hline Acetyl carbonyl & 169.71 & 169.6 \\
\hline Acetyl methyl & 21.10 & 21.0 \\
\hline
\end{tabular}

TABLE 3: NMR data for compound 2 in DMSO- $d_{6}$.

\begin{tabular}{|c|c|c|}
\hline C. no. & ${ }^{13} \mathrm{C}$ NMR $(101 \mathrm{MHz}) \delta$ & ${ }^{1} \mathrm{H}$ NMR $(400 \mathrm{MHz}) \delta$ \\
\hline 2 & $155.37(\mathrm{~S})$ & - \\
\hline 3 & $133.17(\mathrm{~S})$ & - \\
\hline 4 & $177.50(\mathrm{~S})$ & - \\
\hline 5 & $156.28(\mathrm{~S})$ & - \\
\hline 6 & $93.47(\mathrm{D})$ & $6.40(\mathrm{~d}, J=2.1 \mathrm{~Hz}, 1 \mathrm{H})$ \\
\hline 7 & $164.09(\mathrm{~S})$ & - \\
\hline 8 & $98.70(\mathrm{D})$ & $6.19(\mathrm{~d}, J=2.0 \mathrm{~Hz}, 1 \mathrm{H})$ \\
\hline 9 & $161.34(\mathrm{~S})$ & - \\
\hline 10 & $103.95(\mathrm{~S})$ & - \\
\hline $1^{\prime}$ & $121.27(\mathrm{~S})$ & - \\
\hline $2^{\prime}$ & $122.36(\mathrm{D})$ & $7.76(\mathrm{dd}, J=8.5,2.2 \mathrm{~Hz}, 1 \mathrm{H})$ \\
\hline $3^{\prime}$ & $115.30(\mathrm{D})$ & $6.83(\mathrm{~d}, J=8.5 \mathrm{~Hz}, 1 \mathrm{H})$ \\
\hline $4^{\prime}$ & $148.57(\mathrm{~S})$ & - \\
\hline $5^{\prime}$ & $145.03(\mathrm{~S})$ & - \\
\hline $6^{\prime}$ & $115.87(\mathrm{D})$ & $7.53(\mathrm{~d}, J=2.3 \mathrm{~Hz}, 1 \mathrm{H})$ \\
\hline $1^{\prime \prime}$ & 104.73 (D) & $4.56(\mathrm{~d}, J=7.2 \mathrm{~Hz}, 1 \mathrm{H})$ \\
\hline $2^{\prime \prime}$ & $73.71(\mathrm{D})$ & - \\
\hline $3^{\prime \prime}$ & $69.50(\mathrm{D})$ & - \\
\hline $4^{\prime \prime}$ & 75.97 (D) & - \\
\hline $5^{\prime \prime}$ & $76.28(\mathrm{D})$ & - \\
\hline $6^{\prime \prime}$ & $65.73(\mathrm{~T})$ & - \\
\hline $1^{\prime \prime \prime}$ & $98.43(\mathrm{D})$ & $5.70(\mathrm{~d}, J=7.6 \mathrm{~Hz}, 1 \mathrm{H})$ \\
\hline $2^{\prime \prime \prime}$ & 79.90 (D) & - \\
\hline $3^{\prime \prime \prime}$ & $67.85(\mathrm{D})$ & - \\
\hline $4^{\prime \prime \prime}$ & $74.02(\mathrm{D})$ & - \\
\hline $5^{\prime \prime \prime}$ & $60.07(\mathrm{~T})$ & - \\
\hline
\end{tabular}


TABLE 4: NMR data for compound 3 in DMSO- $d_{6}$.

\begin{tabular}{lcc}
\hline C. no. & ${ }^{13} \mathrm{C} \mathrm{NMR}(101 \mathrm{MHz}) \delta$ & ${ }^{1} \mathrm{H} \mathrm{NMR}(400 \mathrm{MHz}) \delta$ \\
\hline 2 & 156.34 & - \\
3 & 133.27 & - \\
4 & 177.53 & - \\
5 & 156.49 & $6.20(\mathrm{~d}, J=2.1 \mathrm{~Hz}, 1 \mathrm{H})$ \\
6 & 98.84 & - \\
7 & 164.59 & $6.43(\mathrm{~d}, J=2.0 \mathrm{~Hz}, 1 \mathrm{H})$ \\
8 & 93.79 & - \\
9 & 161.29 & - \\
10 & 104.01 & - \\
$1^{\prime}$ & 120.99 & $8.05(\mathrm{~d}, J=8.0 \mathrm{~Hz}, 2 \mathrm{H})$ \\
$2^{\prime}$ & 130.97 & $6.87(\mathrm{t}, J=8.0 \mathrm{~Hz}, 2 \mathrm{H})$ \\
$3^{\prime}$ & 115.20 & - \\
$4^{\prime}$ & 160.03 & $6.87(\mathrm{t}, J=8.0 \mathrm{~Hz}, 2 \mathrm{H})$ \\
$5^{\prime}$ & 115.20 & $8.05(\mathrm{~d}, J=8.0 \mathrm{~Hz}, 2 \mathrm{H})$ \\
$6^{\prime}$ & 130.97 & $5.45(\mathrm{~d}, J=7.5 \mathrm{~Hz}, 1 \mathrm{H})$ \\
$1^{\prime \prime}$ & 100.98 & 3.18 \\
$2^{\prime \prime}$ & 74.30 & $3.09(\mathrm{~d}, J=5.0 \mathrm{~Hz}, 2 \mathrm{H})$ \\
$3^{\prime \prime}$ & 77.54 & $3.09(\mathrm{~d}, J=5.0 \mathrm{~Hz}, 2 \mathrm{H})$ \\
$4^{\prime \prime}$ & 69.98 & 3.21 \\
$5^{\prime \prime}$ & 76.50 & $3.56,3.32$ \\
$6^{\prime \prime}$ & 60.93 & \\
\hline
\end{tabular}

TABLE 5: NMR data for compound 4 in DMSO- $d_{6}$.

\begin{tabular}{lcr}
\hline C. no. & ${ }^{13} \mathrm{C} \mathrm{NMR}(101 \mathrm{MHz}) \delta$ & ${ }^{1} \mathrm{H} \mathrm{NMR}(400 \mathrm{MHz}) \delta$ \\
\hline 2 & $81.05(\mathrm{D})$ & $4.49(\mathrm{~d}, J=7.4 \mathrm{~Hz}, 1 \mathrm{H})$ \\
3 & $66.38(\mathrm{D})$ & $3.83(\mathrm{td}, J=7.7,5.4 \mathrm{~Hz}, 1 \mathrm{H})$ \\
4 & $27.89(\mathrm{~T})$ & $2.66(\mathrm{dd}, J=16.0,5.4 \mathrm{~Hz}, 1 \mathrm{H})$ \\
$2.36(\mathrm{dd}, J=16.0,8.0 \mathrm{~Hz}, 1 \mathrm{H})$ & $99.13(\mathrm{~S})$ & - \\
5 & $156.23(\mathrm{~S})$ & - \\
6 & $95.19(\mathrm{D})$ & $5.89(\mathrm{~d}, J=2.0 \mathrm{~Hz}, 1 \mathrm{H})$ \\
7 & $156.51(\mathrm{~S})$ & - \\
8 & $93.92(\mathrm{D})$ & $5.70(\mathrm{~d}, J=2.0 \mathrm{~Hz}, 1 \mathrm{H})$ \\
9 & $155.41(\mathrm{~S})$ & - \\
10 & $130.67(\mathrm{~S})$ & - \\
$1^{\prime}$ & $114.58(\mathrm{D})$ & $6.73(\mathrm{~d}, J=2.0 \mathrm{~Hz}, 1 \mathrm{H})$ \\
$2^{\prime}$ & $144.90(\mathrm{~S})$ & - \\
$3^{\prime}$ & $144.90(\mathrm{~S})$ & - \\
$4^{\prime}$ & $115.15(\mathrm{D})$ & $6.69(\mathrm{~d}, J=8.0 \mathrm{~Hz}, 1 \mathrm{H})$ \\
$5^{\prime}$ & $118.49(\mathrm{D})$ & $6.60(\mathrm{dd}, J=8.0,2.0 \mathrm{~Hz}, 1 \mathrm{H})$ \\
$6^{\prime}$ & &
\end{tabular}

quercetin-3-O- $\alpha$-arabinofuranosyl- $\beta$-D-galactopyranoside. Main fraction 7 (1.00 g) was loaded onto a Sephadex LH-20 column and eluted with $\mathrm{MeOH}$; yielded seven subfractions were collected and subfractions 2 and 4 were identified as fructose and glucose, respectively. A Bruker mode AMX 500 NMR and 400 NMR spectrometer operating and a standard pulse system collected ${ }^{1} \mathrm{H}$ and ${ }^{13} \mathrm{C}$ NMR spectra. The instrument ran at 500 and $400 \mathrm{MHz}$ in ${ }^{1} \mathrm{H}$ and 125 to $100 \mathrm{MHz}$ in ${ }^{13} \mathrm{C}$, and DMSO- $\mathrm{d}_{6}$ and $\mathrm{CD}_{3} \mathrm{OD}$ were used as solvents.

2.3. Antimicrobial and Antiplasmodial Activities. The extract and isolated compounds were screened for antimicrobial and antiplasmodial activities using the reported methods by $[8,9]$.
2.4. Obtaining Essential Oil. To extract an essential oil, plant material was chopped into small pieces (3-4 mm). Chopped plant material (535.36 g) was subjected to steam-distillation using $1 \mathrm{~L}$ of deionized water on true steam distiller with a $1 \mathrm{~L}$ capacity for 2.5 hours. Essential oil transferred to GS vial by using $0.5 \mathrm{ml}$ of pentane. The oil yields based on dry weight of samples were $0.1-1.5 \%$. The collected essential oil was analyzed by GC-MS-FID on an Agilent 7890A GC system inert XL MSD with triple-axis detector and Agilent 7693 autosampler. GC was equipped with a DB-5 fused silica capillary column $(30 \mathrm{~m} \times 0.25 \mathrm{~mm}$, film thickness of $0.25 \mathrm{~mm}$ ). The injector temperature was $240^{\circ} \mathrm{C}$ and the column temperature was initiated at $60^{\circ} \mathrm{C}$, increased at $3^{\circ} \mathrm{C} /$ $\min$ to $240^{\circ} \mathrm{C}$, and held for $5 \mathrm{~min}$. The carrier gas was $\mathrm{He}$ and the injection volume was $5 \mu \mathrm{L}$ (splitless). The MS 
TABLE 6: Antimicrobial activity results of extract and isolated compounds.

\begin{tabular}{|c|c|c|c|c|c|c|c|c|c|}
\hline $\begin{array}{l}\text { Sample } \\
\text { code }\end{array}$ & $\begin{array}{c}\text { Candida } \\
\text { albicans } \text { Pinh }\end{array}$ & $\begin{array}{c}\text { Aspergillus } \\
\text { fumigatus Pinh }\end{array}$ & $\begin{array}{c}\text { Cryptococcus } \\
\text { neoformans Pinh }\end{array}$ & $\begin{array}{l}\text { MRS } \\
\text { Pinh }\end{array}$ & $\begin{array}{l}\text { E. coli } \\
\text { Pinh }\end{array}$ & $\begin{array}{l}\text { Pseudomonas } \\
\text { aeruginosa Pinh }\end{array}$ & $\begin{array}{c}\text { Kp } \\
\text { Pinh } \\
\end{array}$ & $\begin{array}{l}\text { VRE } \\
\text { Pinh }\end{array}$ & Test conc. \\
\hline FLU & $<0.1$ & $>100$ & $<0.1$ & $>100$ & $>100$ & $>100$ & $>100$ & $>100$ & $\begin{array}{c}100-4 \mu \mathrm{g} / \\
\mathrm{mL}\end{array}$ \\
\hline $\mathrm{AMB}$ & 0.176 & 0.261 & 0.251 & $>100$ & $>100$ & $>100$ & $>100$ & $>100$ & $\begin{array}{c}100-4 \mu \mathrm{g} / \\
\mathrm{mL}\end{array}$ \\
\hline CIPRO & $>10$ & $>10$ & $>10$ & $>10$ & $<0.01$ & 0.3 & $>10$ & $>10$ & $\begin{array}{c}10-0.4 \mu \mathrm{g} / \\
\mathrm{mL}\end{array}$ \\
\hline Vanco & $>100$ & $>100$ & $>100$ & $<0.1$ & 79.864 & $>100$ & $>100$ & $>100$ & $\begin{array}{c}100-4 \mu \mathrm{g} / \\
\mathrm{mL}\end{array}$ \\
\hline METH & $>100$ & $>100$ & $>100$ & 16.096 & $>100$ & 73.476 & $>100$ & $>100$ & $\begin{array}{c}100-4 \mu \mathrm{g} / \\
\mathrm{mL}\end{array}$ \\
\hline CEFO & $>100$ & $>100$ & $<0.097$ & 1.136 & $>100$ & 1.113 & $>100$ & $>100$ & $\begin{array}{c}100-4 \mu \mathrm{g} / \\
\mathrm{mL}\end{array}$ \\
\hline MERO & $>100$ & $>100$ & $>100$ & $<0.1$ & 13.738 & 6.797 & 37.76 & $>100$ & $\begin{array}{c}100-4 \mu \mathrm{g} / \\
\mathrm{mL}\end{array}$ \\
\hline Extract & $>200$ & $>200$ & $>200$ & & $>200$ & $>200$ & $>200$ & $>200$ & $>200$ \\
\hline 1 & $>20$ & $>20$ & $>20$ & $>20$ & $>20$ & $>20$ & $>20$ & $>20$ & $\begin{array}{c}20-0.8 \mu \mathrm{g} / \\
\mathrm{mL}\end{array}$ \\
\hline 2 & $>20$ & $>20$ & $>20$ & $>20$ & $>20$ & $>20$ & $>20$ & $>20$ & $\begin{array}{c}20-0.8 \mu \mathrm{g} / \\
\mathrm{mL}\end{array}$ \\
\hline 3 & $>20$ & $>20$ & $>20$ & $>20$ & $>20$ & $>20$ & $>20$ & $>20$ & $\begin{array}{c}20-0.8 \mu \mathrm{g} / \\
\mathrm{mL}\end{array}$ \\
\hline 4 & $>20$ & $>20$ & $>20$ & $>20$ & $>20$ & $>20$ & $>20$ & $>20$ & $\begin{array}{c}20-0.8 \mu \mathrm{g} / \\
\mathrm{mL}\end{array}$ \\
\hline 5 & $>20$ & $>20$ & $>20$ & $>20$ & $>20$ & $>20$ & $>20$ & $>20$ & $\begin{array}{c}20-0.8 \mu \mathrm{g} / \\
\mathrm{mL}\end{array}$ \\
\hline
\end{tabular}

FLU: fluconazole, AMB: amphotericin B (New Lot), CIPRO: ciprofloxacin (New Lot), Vanco: vancomycin, METH: methicillin, CEFO: cefotaxime, MERO: meropenem, VRE: vancomycin-resistant enterococci, and MRSA: methicillin-resistant S. aureus.

TABLe 7: Antiplasmodial activity results of extract and isolated compounds.

\begin{tabular}{|c|c|c|c|c|c|c|}
\hline $\begin{array}{l}\text { Sample } \\
\text { code }\end{array}$ & $\begin{array}{l}\text { P. falciparum D6 } \\
\text { IC50 }\end{array}$ & P. falciparum D6 SI & $\begin{array}{l}\text { P. falciparum W2 } \\
\text { IC50 }\end{array}$ & $\begin{array}{l}\text { P. falciparum W2 } \\
\text { SI }\end{array}$ & $\begin{array}{l}\text { VERO } \\
\text { IC50 }\end{array}$ & Test conc. \\
\hline ART & $<26.4$ & $>9$ & $<26.4$ & $>9$ & $>238$ & $238-26.4 \mathrm{ng} / \mathrm{mL}$ \\
\hline CQ & $<26.4$ & $>9$ & 213.7 & $>1.1$ & $>238$ & $238-26.4 \mathrm{ng} / \mathrm{mL}$ \\
\hline Extract & $>47600$ & 1 & $>47600$ & 1 & $>47600$ & $\begin{array}{c}47600-5288.9 \mathrm{ng} / \\
\mathrm{mL}\end{array}$ \\
\hline 1 & $>4760$ & 1 & $>4760$ & 1 & $>4760$ & $4760-528.9 \mathrm{ng} / \mathrm{mL}$ \\
\hline 2 & $>4760$ & 1 & $>4760$ & 1 & $>4760$ & $4760-528.9 \mathrm{ng} / \mathrm{mL}$ \\
\hline 3 & $>4760$ & 1 & $>4760$ & 1 & $>4760$ & $4760-528.9 \mathrm{ng} / \mathrm{mL}$ \\
\hline 4 & $>4760$ & 1 & $>4760$ & 1 & $>4760$ & $4760-528.9 \mathrm{ng} / \mathrm{mL}$ \\
\hline 5 & $>4760$ & 1 & $>4760$ & 1 & $>4760$ & $4760-528.9 \mathrm{ng} / \mathrm{mL}$ \\
\hline
\end{tabular}

ART: artemisinin, CQ: chloroquine.

TABle 8: Constituent composition of essential oil from Agrimonia asiatica.

\begin{tabular}{|c|c|c|c|c|}
\hline No. & Constituents & $\mathrm{RT}^{*}$ & $\mathrm{KI}^{*}$ & $\%$ \\
\hline 1 & 2-Pentylfuran & 7.594 & 970.28 & 1.3 \\
\hline 2 & Nonanal & 11.634 & 1107.20 & 4.2 \\
\hline 3 & 6,10-Dimethyl-5, 9-undecadien-2-one & 26.205 & 1450.26 & 3.8 \\
\hline 4 & $\beta$-Selinene & 27.537 & 1485.46 & 36.4 \\
\hline 5 & $\beta$-Guaiene & 27.893 & 1494.58 & 2.4 \\
\hline 6 & Germacrene A & 28.514 & 1510.21 & 2.5 \\
\hline 7 & $\alpha$-Panasinsene & 28.889 & 1519.49 & 21.7 \\
\hline 8 & Z-Nuciferol acetate & 40.395 & 1829.33 & 3.2 \\
\hline 9 & 7-Hydroxycoumarin & 40.713 & 1839.58 & 5.7 \\
\hline 10 & Palmitic acid & 44.718 & 1961.84 & 7.8 \\
\hline 11 & 4-Methyl-2-4-bis (4-trimethylsilixphenylpentene-1) & 49.878 & 2130.60 & 4.8 \\
\hline 12 & 1,2 -Nonadiene & 50.073 & 2136.97 & 6.2 \\
\hline Total & & & & 100 \\
\hline
\end{tabular}

$\mathrm{RT}^{*}$ : retention time, $\mathrm{KI}^{*}$ : Kovats index. 
conditions were mass range of $50-550 \mathrm{~m} / z$, filament delay of $3.50 \mathrm{~min}$. The MS conditions were mass range of $50-550 \mathrm{~m} / z$, filament delay of $3.50 \mathrm{~min}$. The oil components were identified by comparing their mass spectra with the NIST Library as well as with authentic compounds. This was confirmed by comparison of their retention indices with those of authentic compounds as well as with data published in the literature.

\section{Result and Discussion}

Phytochemical investigation on ethanolic extract of Agrimonia asiatica led to isolation of four flavonoid derivatives. All the isolated compounds were identified by analyzing their spectral data analysis as follows: 3-O-kaempherol 2,3-di-Oacetyl-4-O-(cis-p-coumaroyl)-6-O-(trans-p-coumaroyl)- $\beta$-Dglucosopyranoside (1), quercetin-3-O- $\alpha$-arabinofuranosyl$\beta$-D-galactopyranoside (2), kaempferol-3-glycoside (3), catechin (4), and sucrose (5) (see Tables 1-5 and their NMR spectra given in Supplementary Materials Figures 1-19).

All the extracts, fractions, and isolated compounds were tested for antimicrobial and antiplasmodial activities. None of the extracts and isolates exhibited significant activity (see Tables 6 and 7).

The oil components were identified by comparing their mass spectra with the NIST Library as well as with authentic compounds. This was confirmed by comparison of their retention indices with those of authentic compounds as well as with data published in the literature [14].

The identified compounds, their retention indices, and their percentage compositions were summarized in Table 8 . The constituents are arranged in order according to their elution on DB- 5 column. The oil yields based on dry weight of samples were $0.003-0.004 \%(\mathrm{~W} / \mathrm{W})$. The major components of oil were found to be $\beta$-selinene (36.4\%), $\alpha$-panasinsene (21.7\%), palmitic acid $(7.8 \%), 1,2$-nonadiene $(6.2 \%)$, nonanal (4.2\%), germacrene $\mathrm{A}(2.5 \%)$, and $\beta$-guaiene (2.4\%).

\section{Conclusion}

To sum up, chromatographic purification of ethanolic extract over Silica gel yielded five compounds: 3-O-kaempherol 2,3- di-O-acetyl-4-O-(cis-p-coumaroyl)-6-O-(transp-coumaroyl)- $\beta$-D-glucosopyranoside (1), quercetin-3-O$\alpha$-arabinofuranosyl- $\beta$-D-galactopyranoside (2), kaempferol3 -glycoside (3), catechin (4), and sucrose (5). Compounds 1 and 2 were first time to be reported from this plant. Screening results of antimicrobial and antiplasmodial activities did not show any positive results. We found that the major components of Agrimonia asiatica essential oil are $\beta$-selinene (36.4\%), $\alpha$-panasinsene (21.7\%), palmitic acid (7.8\%), 1,2-nonadiene (6.2\%), nonanal (4.2\%), germacrene A $(2.5 \%)$, and $\beta$-guaiene (2.4\%).

\section{Data Availability}

The data used to support the findings of this study are included within the article.

\section{Conflicts of Interest}

The authors declare that there are no conflicts of interest regarding the publication of this paper.

\section{Acknowledgments}

The authors are grateful for the financial support of Kazakhstan Government and the National Center for Natural Product Research, University of Mississippi and in part by the USDA Agricultural Research Service Specific Cooperative Agreement no. 58-6060-6-015. The authors wish to thank Drs. Charles L. Cantrell and Green Solomon USDA-ARS-NPURU for GC-MS results.

\section{Supplementary Materials}

Figure 1: ${ }^{1} \mathrm{H}$ NMR spectrum of 3-O-kaempherol 2,3-di-Oacetyl-4-O-(cis-p-coumaroyl)-6-O-(trans-p-coumaroyl)$\beta$-D-glucosopyranoside. Figure $2:{ }^{13} \mathrm{C}$ NMR spectrum of 3-O-kaempherol 2,3-di-O-acetyl-4-O-(cis-p-coumaroyl)-6-O(trans-p-coumaroyl)- $\beta$-D-glucosopyranoside. Figure 3: EPT 135 spectrum of 3-O-kaempherol 2,3-di-O-acetyl-4-O-(cis-pcoumaroyl)-6-O-(trans-p-coumaroyl)- $\beta$-D-glucosopyranoside. Figure 4: HSQC spectrum of 3-O-kaempherol 2,3-di-O-acetyl4-O-(cis-p-coumaroyl)-6-O-(trans-p-coumaroyl)- $\beta$-D-glucosopyranoside. Figure 5: HMBC spectrum of 3-O-kaempherol 2,3-di-O-acetyl-4-O-(cis-p-coumaroyl)-6-O-(transp-coumaroyl)- $\beta$-D-glucosopyranoside. Figure 6: COSY spectrum of 3-O-kaempherol 2,3-di-O-acetyl-4-O-(cis-pcoumaroyl)-6-O-(trans-p-coumaroyl)- $\beta$ - $\mathrm{D}$-glucosopyranoside. Figure 7: ${ }^{1} \mathrm{H}$ NMR spectrum of quercetin-3-O$\alpha$-arabinofuranosyl- $\beta$-D-galactopyranoside. Figure $8:{ }^{13} \mathrm{C}$ NMR spectrum of quercetin-3-O- $\alpha$-arabinofuranosyl- $\beta$-Dgalactopyranoside. Figure 9: DEPT 135 spectrum of quercetin-3-O- $\alpha$-arabinofuranosyl- $\beta$-D-galactopyranoside. Figure 10 : HSQC spectrum of quercetin-3-O- $\alpha$-arabinofuranosyl- $\beta$-D-galactopyranoside. Figure 11: HMBC spectrum of quercetin-3-O$\alpha$-arabinofuranosyl- $\beta$-D-galactopyranoside. Figure $12:{ }^{1} \mathrm{H}$ NMR spectrum of kaempferol-3-glycoside. Figure 13: ${ }^{13} \mathrm{C}$ NMR spectrum of kaempferol-3-glycoside. Figure 14: DEPT 135 spectrum of kaempferol-3-glycoside. Figure 15: HSQC spectrum of kaempferol-3-glycoside. Figure 16: HMBC spectrum of kaempferol-3-glycoside. Figure 17: ${ }^{1} \mathrm{H}$ NMR spectrum of catechin. Figure 18: ${ }^{13} \mathrm{C}$ NMR spectrum of catechin. Figure 19: DEPT 135 spectrum of catechin. (Supplementary Materials)

\section{References}

[1] M. Kostryca and M. Chwil, "Biologically active compounds in Agrimonia eupatoria L. and their therapeutic effects," World Scientific News, vol. 89, pp. 90-97, 2017.

[2] O. A. Sapko, O. V. Chebonenko, A. S. Utarbaeva, A. Z. Amirkulova, and A. K. Tursunova, "Antioxidant activity of medicinal plants from southeastern Kazakhstan," Pharmaceutical Chemistry Journal, vol. 50, no. 9, pp. 603-607, 2016.

[3] O. A. Sapko, A. K. Tursunova, A. O. Abaildaev, O. V. Chebonenko, A. V. Krasnoshtanov, and A. S. Utarbaeva, "Effects of extracts of Agrimonia asiatica and Geranium 
collinum on lipid peroxidation and the blood antioxidant enzyme activity in rats with alloxan diabetes," Pharmaceutical Chemistry Journal, vol. 51, no. 7, pp. 596-601, 2017.

[4] M. Mehdi and N. N. Mehrdokht, "Chemical composition of the essential oils extracted from the leaf and flowers of Marsdenia erecta (L.) R. Br. in Iran," Journal of Essential Oil Bearing Plants, vol. 12, no. 1, pp. 87-91, 2009.

[5] H. Wang, Y. Liu, S. Wei, Z. Yan, and X. Jin, "Comparative chemical composition of the essential oils obtained by microwave-assisted hydrodistillation and hydrodistillation from Agrimonia pilosa LEDEB. Collected in three different regions of China," Chemistry \& Biodiversity, vol. 9, no. 3, pp. 662-668, 2012.

[6] R. Srivedavyasasri, K. A. Zhaparkulova, Z. B. Sakipova, L. Ibragimova, and S. A. Ross, "Phytochemical and biological studies on Ziziphora bungeana," Chemistry of Natural Compounds, vol. 54, no. 1, pp. 195-197, 2018.

[7] D. M. Grochowski, K. Skalicka-Woźniak, I. E. Orhan et al., "A comprehensive review of agrimoniin," Annals of the New York Academy of Sciences, vol. 1401, no. 1, pp. 166-180, 2017.

[8] S. B. Bharate, S. I. Khan, N. A. M. Yunus et al., "Antiprotozoal and antimicrobial activities of O-alkylated and formylated acylphloroglucinols," Bioorganic \& Medicinal Chemistry, vol. 15, no. 1, pp. 87-96, 2007.

[9] S. Manohar, M. Tripathi, and D. Rawat, "4-Aminoquinoline based molecular hibrids as antimalarials: an overview," Current Topics in Medicinal Chemistry, vol. 14, no. 14, pp. 1706-1733, 2014.

[10] Repub Korean Kongkae Taeho Kongbo (2012), KR 2012090262 A 20120817.

[11] S. Y. Lee, Y. J. Shin, S. U. Choi, and K. R. Lee, "A new flavonol glycoside from the aerial part of Rudbeckia laciniata," Archives of Pharmacal Research, vol. 37, no. 7, pp. 834-838, 2014.

[12] T. Okuyama, K. Hosoyama, Y. Hiraga, G. Kurono, and T. Takemoto, "The constituents of Osmunda spp. II. A new flavonol glycoside of Osmunda asiatica," Chemical \& Pharmaceutical Bulletin, vol. 26, no. 10, pp. 3071-3074, 1978.

[13] M. H. Abd-El-Razek, "NMR assignments of four catechin epimers," Asian Journal of Chemistry, vol. 19, no. 6, pp. 4867-4872, 2007.

[14] R. P. Adams, Identification of Essential Oil Components by Gas Chromatography/Quadrupole Mass Spectroscopy, Allured Publishing Corporation, Carol Stream, IL, USA, 2001. 